\title{
Coreopsis Tinctoria Modulates Lipid Metabolism by Decreasing Low-Density Lipoprotein and Improving Gut Microbiota
}

\author{
Zhigang Ren ${ }^{\mathrm{a} e \mathrm{e}}$ Yali Lib,h Jiangyun Liư Haitao Lid,h Ang Li ${ }^{\mathrm{c}}$ \\ Liangjie Hong ${ }^{f}$ Guangying Cui ${ }^{\mathrm{a}, \mathrm{e}}$ Ranran Sun ${ }^{\mathrm{a}, \mathrm{e}}$ Muhuyati Wulasihan ${ }^{\mathrm{b}, \mathrm{h}}$ \\ Junhui Sun ${ }^{f}$ Yujun Song ${ }^{g}$ Zujiang Yu ${ }^{a, e} \quad$ Xinhua Chen ${ }^{f}$
}

\begin{abstract}
aDepartment of Infectious Diseases, the First Affiliated Hospital of Zhengzhou University, Zhengzhou, ${ }^{b}$ Department of Internal Medicine, the First Affiliated Hospital of Xinjiang Medical University, Urumqi, 'College of Pharmaceutical Sciences, Soochow University, Suzhou, ${ }^{\mathrm{d} A b d o m i n a l ~ S u r g e r y ~ \& ~ O n c o l o g y ~}$ Department, Changji Branch of the First Affiliated Hospital of Xinjiang Medical University, Changji, ePrecision Medicine Center, the First Affiliated Hospital of Zhengzhou University, Zhengzhou, fDepartment of Hepatobiliary and Pancreatic Surgery, the First Affiliated Hospital, School of Medicine, Zhejiang University, Key Laboratory of Combined Multi-organ Transplantation, Ministry of Public Health, Hangzhou, ${ }^{9 B}$ Beijing Key Laboratory for Magneto-Photoelectrical Composite and Interface Science, School of Mathematics and Physics, Center for Modern Physics Technology, Science and Technology University of Beijing, Beijing, 'State Key Laboratory of Pathogenesis, Prevention, Treatment of High Incidence Diseases in Central Asia, the First Affiliated Hospital of Xinjiang Medical University, Urumqi, China
\end{abstract}

\section{Key Words}

Coreopsis tinctoria $\cdot$ Hyperlipidemia $\bullet$ Gut microbiota

\begin{abstract}
Background/Aims: The prevalence of hyperlipidemia is increasing rapidly. The role of Coreopsis tinctoria (CT) in amending lipid metabolism in hyperlipidemia patients has not been reported. This study aims to evaluate the role of $\mathrm{CT}$ in altering lipid metabolism in hyperlipidemia patients and to explore the possible mechanisms mediated by gut microbiota in hyperlipidemia mice models. Methods: A retrospective analysis in 40 hyperlipidemia patients was conducted, in which 20 patients took fenofibrate and another 20 patients normatively drank water with CT. Hyperlipidemia mice models were also established. Blood biochemical tests were performed using an automatic biochemical analyzer. Liver histopathology was observed by hematoxylin and eosin staining. Ileocecal samples were collected from mice, and bacterial DNA was extracted and sequenced by MiSeq sequencing. Bacterial composition and differences were analyzed. Results: In hyperlipidemia patients, CT was associated with decreased triglyceride and low-density lipoprotein (LDL) levels without liver injury. The experimental hyperlipidemia model also verified a similar result. Gut microbial richness and diversity were significantly

Z. Ren and Y. Li contributed equally to this work.

\begin{tabular}{ll}
\hline $\begin{array}{l}\text { Zujiang Yu } \\
\text { and Xinhua Chen }\end{array}$ & Department of Infectious Diseases, the First Affiliated Hospital of Zhengzhou University, Zhengzhou 450052; \\
& Department of Hepatobiliary and Pancreatic Surgery, the First Affiliated Hospital, School of Medicine, Zhejiang \\
& University, Hangzhou, 310003 (China) E-Mail johnyuem@zzu.edu.cn, xinhua_chen@zju.edu.cn
\end{tabular}
\end{abstract}


decreased in hyperlipidemic mice, but increased after CT treatment. Bacterial communities were significantly differentiated between normal controls and hyperlipidemic mice. CT administration improved gut microbiota composition to an approximately normal status. Meanwhile, CT administration attenuated bacterial alterations at the class, order, family, and genus levels in hyperlipidemic mice. Importantly, the genera Barnesiella, Lactobacillus, and Helicobacter achieved high discriminatory power in hyperlipidemic mice relative to normal controls. Conclusions: CT can modulate blood lipid metabolism with improvement of liver function by decreasing LDL and improving gut microbiota compositions. These findings may provide novel therapeutic strategies for patients with hyperlipidemia.

\section{(C) 2018 The Author(s)}

Published by S. Karger AG, Basel

\section{Introduction}

The prevalence of hyperlipidemia, which has associated risks of end-stage liver and cardiovascular diseases, is increasing rapidly along with the growing prevalence of obesity over the last several decades $[1,2]$. Substantial studies have indicated that hyperlipidemia is an independent risk factor for obesity, coronary heart disease [3, 4], stroke [5], and sudden cardiac death [6]. It is also closely associated with the occurrence of non-alcoholic fatty liver disease, a condition characterized by excessive fat accumulation in the form of triglycerides $[1,2]$, which dramatically increases the risks of cirrhosis, liver failure, and hepatocellular carcinoma [7]. As a common lipid-reducing drug, fenofibrate can reduce cholesterol and triglycerides in the blood effectively. However, the long-term use of fenofibrate can cause liver damage [8]. Thus, it is crucial to identify effective and safe therapies to reduce blood lipids and cholesterol.

Coreopsis tinctoria (CT) is a plant widely cultivated in western China and the Midwest region of the United States. Because its growth environment is less polluted and occurs at high altitudes, CT contains many kinds of compounds that benefit human health, and has gradually become popular as a type of tea drink. Our previous study indicated that the flavonoids extracted from CT show anti-hyperlipidemic effects, particularly in lowering triglycerides, reducing lipid deposition, and protecting liver function in an animal model [9]. Some pharmacological studies have also reported that CT is rich in flavonoids and can adjust glucose metabolism, promote blood circulation, and reduce cardiovascular disease risk [10, 11]. Thus, a retrospective analysis was performed in patients with hyperlipidemia in this study, and we validated a protective role of CT in patients with hyperlipidemia.

Human gut microbiotas have been well recognized as the most important microecosystem mediating a symbiotic relationship with other organs [12-16]. Gut microbial alterations contribute to the onset and progression of non-alcoholic fatty liver disease $[17,18]$ and cardiovascular disease [19]. Therefore, we speculated that the beneficial role of CT on hyperlipidemia might be partially attributed to the regulation of gut microbiota. Furthermore, we established a mouse model of hyperlipidemia and explored the potential mechanisms by which CT impacts hyperlipidemia with respect to gut microbial alterations.

\section{Materials and Methods}

A retrospective analysis of patients with hyperlipidemia

In our previous study, we identified the chemical composition of CT extracts using a high-performance liquid chromatography/photodiode array system [9]. The constituent compounds are mainly aglycones and glycosides of the flavanone/chalcone flavonoid type. The major 12 compounds include $3,4^{\prime}, 5$, 6,7-pentahydroxyflavanone- $O$-hexoside, chlorogenic acid, flavanomarein, flavanokanin, quercetagitin-7$O$-glucoside, 3, 4',5, 6,7-pentahydroxyflavanone, 3',5, 5',7-tetrahydroxyflavanone- $O$-hexoside, marein, 3',5, $5^{\prime}, 7$-tetrahydroxy flavanone, okanin, dicaffeoylquinic acid, and coreopsin.

Because CT has gradually become popular as a type of tea drink, many people drink tea with CT regularly. Accordingly, we conducted a retrospective analysis of patients with hyperlipidemia. Hyperlipidemia was 


\section{Cellular Physiology Cell Physiol Biochem 2018;48:1060-1074 \begin{tabular}{l|l} 
and Biochemistry Published 10.1159/000491973 & $\begin{array}{l}\text { D } 2018 \text { The Author(s). Published by S. Karger AG, Basel } \\
\text { www.karger.com/cpb }\end{array}$
\end{tabular} \\ Ren et al.: Coreopsis Tinctoria Modulates Lipids Metabolism}

diagnosed according to serum triglyceride (TG) levels exceeding $6.2 \mathrm{mmol} / \mathrm{L}$ with elevated low-density lipoprotein (LDL, i.e., hyperlipoproteinemia type IIa) in patients. CT samples were collected from Dabanchen (Xinjiang, China; lot number 130501), and the botanical origin of the material was identified by the College of Pharmaceutical Sciences, Shandong Traditional Medical University (Jinan, China).

In this study, patients with hyperlipidemia were excluded if they suffered from allergies, liver disease, kidney disease, gallbladder disease, diabetes, heart disease, or thyroid disorder. We retrospectively analyzed a total of 40 patients with hyperlipidemia, in which 20 patients took fenofibrate orally at $145 \mathrm{mg}$ once a day, and another 20 patients normatively drank water with CT (100 mg/kg/day). Meanwhile, 20 healthy volunteers who drink tea with CT regularly were utilized as a healthy control group. In all healthy control individuals, physical examination; liver biochemistry; routine examination of blood, urine, and stools; serological tests (including the detection of hepatitis B surface antigen, hepatitis C virus antibody, Treponema pallidum antibody, and human immunodeficiency virus antibody); liver function; renal function; electrolyte; liver ultrasound; electrocardiogram; and chest X-ray results were within the normal range. Exclusion criteria for healthy volunteers included hypertension, diabetes, obesity, metabolic syndrome, irritable bowel syndrome, nonalcoholic fatty liver disease, coeliac disease, and liver cirrhosis.

For all participants, clinical information including body mass index (BMI), liver biochemistry, routine blood tests, liver function, and renal function were assessed before drug treatment and 3 months after drug treatment. All participants provided written informed consent upon enrolment. The study conformed to the ethical guidelines of the 1975 Declaration of Helsinki and was approved by the Institutional Review Board of the First Affiliated Hospital, Xinjiang Medical University and the First Affiliated Hospital of Zhengzhou University.

\section{Establishment and study of a mouse model of hyperlipidemia}

A total of 48 specific pathogen-free male mice $(22 \pm 2 \mathrm{~g})$ were housed in the Laboratory Animals Center of the First Affiliated Hospital of Zhengzhou University. The mice were caged in $21^{\circ} \mathrm{C}, 12$-h light/dark cycle conditions and provided sterilized standard rat chow and water.

The mouse model of hyperlipidemia was generated by oral feeding of a high-fat diet $20 \%$ lard, $10 \%$ cholesterol, $2 \%$ bile salts, $0.2 \%$ propylthiouracil, $20 \%$ propylene glycol, $20 \%$ polysorbate- $80 ; 0.2$ $\mathrm{mg} /$ per $10 \mathrm{~g}$ weight per day) for 2 weeks [9]. The hyperlipidemia model was confirmed by blood lipid tests after continuous feeding for 2 weeks. The confirmed hyperlipidemic mice were randomly divided into two groups ( $n=16$ in each group). In the hyperlipidemia (HL) group, the continuous high fat diet was administered for another 6 weeks; for the CT group, the continuous high fat diet and oral feeding at $100 \mathrm{mg} / \mathrm{kg}$ was administered for another 6 weeks. Another 16 mice were fed a normal diet to establish a normal control (NC) group. All procedures were performed according to the "Guide for the Care and Use of Laboratory Animals" published by the National Institutes of Health (NIH publication 86-23, revised 1985). The experimental protocols were approved by the Animal Care and Use Committee of the First Affiliated Hospital of Zhengzhou University.

\section{Sample collection}

After the end-point treatment, all mice were weighed and then sacrificed. Blood samples from the portal vein were collected for blood lipid, routine blood, and liver function tests. Liver specimens in each group were fixed with $10 \%$ buffered formalin for histological examination. The ileocecal fecal contents were collected and immersed in liquid nitrogen, and then stored at $-80^{\circ} \mathrm{C}$ for later gut microbial analysis.

\section{Blood biochemical tests and liver histopathology}

Blood biochemical tests

Blood samples were collected from the portal vein and then centrifuged for $15 \mathrm{~min}$ at $3500 \mathrm{rpm}$ to obtain serum. Serum levels of total cholesterol (TC), triglyceride (TG), high-density lipoprotein (HDL), LDL, total protein, albumin, alanine aminotransferase (ALT), and aspartate aminotransferase (AST) were determined by an automatic biochemical analyzer (Hitachi 7600, Tokyo, Japan).

\section{Liver histopathology}

Liver samples were collected and fixed in $40 \mathrm{~g} / \mathrm{L}$ neutral formaldehyde. After $24 \mathrm{~h}$, liver samples were embedded in paraffin, sectioned into $4-\mu \mathrm{m}$-thick slices and stained with hematoxylin and eosin (H\&E) 


\section{Cellular Physiology Cell Physiol Biochem 2018;48:1060-1074 \\ \begin{tabular}{l|l} 
and Biochemistry Published online: July 24, 2018 & $\begin{array}{l}\text { (c) } 2018 \text { The Author(s). Published by S. Karger AG, Basel } \\
\text { www.karger.com/cpb }\end{array}$ \\
\hline
\end{tabular}}

Ren et al.: Coreopsis Tinctoria Modulates Lipids Metabolism

according to a standard procedure. The H\&E stained samples were observed under light microscopy and judged by two independent pathologists.

\section{Mice ileocecal contents collection and DNA extraction}

The ileocecal content from each mouse was collected for gut microbial analysis. The ileocecal content was divided into two aliquots of $200 \mathrm{mg}$ and immediately stored at $-80^{\circ} \mathrm{C}$. DNA extraction from a frozen aliquot (200 mg) of each fecal sample was performed as previously described [20-22]. DNA concentration was measured using a NanoDrop (Thermo Fisher Scientific, Waltham, MA) and its molecular size was estimated by agarose gel electrophoresis.

\section{PCR amplification and MiSeq sequencing}

The extracted DNA samples were amplified with a set of primers targeting the hypervariable V3-V5 region $(338 \mathrm{~F} / 806 \mathrm{R})$ of the $16 \mathrm{~S}$ rRNA gene. The forward primer was $5^{\prime}$-ACTCCTACGGGAGGCAGCA-3' and the reverse primer was 5'-GGACTACHVGGGTWTCTAAT-3'. PCR amplification was performed as previously described $[20,23]$. DNA libraries were constructed according to the manufacturer's instructions, and sequencing was performed on an IlluminaMiSeq (Illumina, San Diego, CA) platform by Shanghai Itechgene Technology Co. Ltd. (Shanghai, China). The raw reads were deposited into the European Nucleotide Archive database (study accession number, PRJEB15125; secondary study accession number, ERP016828).

\section{Sequence data processing}

According to the specific barcodes, the filtered reads were assigned into different samples, and then the barcodes and primers were trimmed off. The sequences of each library were overlapped using FLASH version 1.2.10 software [24]. Chimeric sequences were detected and removed with UCHIME version 4.2.40 [25] with the $16 \mathrm{~S}$ "golden standard" database provided by the Broad Institute as a reference (version microbiome util-r20110519, http://drive5.com/uchime/gold.fa).

An equal number of random reads were chosen from all samples, and then operational taxonomic units (OTUs) were binned using the UPARSE pipeline [26] with the command "usearch-usearch_global-id 0.97," the identity threshold was set at 0.97 , and an OTU composition table was created. We annotated sequences using RDP classifier version 2.6 [27], with a confidence level set to 0.5 according to the developer's documents (http://rdp.cme.msu.edu/classifier/class_help.jsp\#conf).

\section{Sequence data analysis and statistics}

Bacterial diversity was determined by sampling-based analysis of OTUs and visualized as rarefaction curves using the R statistical computing environment (http://www.R-project.org/) package "vegan." Principal component analysis (PCA) was conducted using the R package to visualize the interactions among bacterial communities of different samples. The weighted unifrac distance and the unweighted unifirac distance were calculated with the phyloseq package [28]. Hellinger distance, Jensen-Shannon distance, and Spearman coefficient distance were calculated with a custom R function provided by EBML (http:// enterotype.embl.de/enterotypes.html\#dm). The specific characterization of fecal microbiota to distinguish toxigenic types was analyzed using a linear discriminant analysis (LDA) effect size (LEfSe) method (http:// huttenhower.sph.harvard.edu/lefse/) for biomarker discovery [29]. Using a normalized relative abundance matrix, LEfSe performs the Kruskal-Wallis rank sum test to determine features with significantly different abundances between assigned taxa and uses LDA to assess the effect size of each feature [30]. The microbial biomarkers were presented at a significance alpha of 0.05 and an effect size threshold of 2 .

\section{Statistical analysis}

Continuous variables were presented as the mean \pm standard error of mean. Student's $t$-tests were used to assess parametric data between both groups. The Wilcoxon rank-sum test was used to evaluate differences in bacterial populations among the three groups. Receiver operating characteristics (ROC) curves were used to define the sensitivity and specificity of bacterial genera to disease diagnosis. Statistical analyses were conducted using SPSS version 19.0 for Windows (SPSS Inc., Chicago, IL). 


\section{Results}

CT alters lipid metabolism by decreasing LDL without liver injury in hyperlipidemia patients

Across the 60 enrolled participants, there were no significant differences in age and sex among the 3 groups (Table 1). Notably, the two groups with hyperlipidemia patients before treatment presented a significantly high BMI compared with the healthy controls (both $p<0.001$, Fig. 1a). Correspondingly, the two groups with hyperlipidemia patients before treatment showed remarkably high TG, low HDL, and high LDL levels in serum compared with healthy controls (all $p<0.001$, Fig. 1b-d). Notably, compared with healthy controls $(2.42 \pm 0.028 \mathrm{mmol} / \mathrm{L})$, serum LDL levels were significantly increased in hyperlipidemia patients before treatment with fenofibrate $\left(3.27 \pm 0.073 \mathrm{mmol} / \mathrm{L}, p=6.55 \times 10^{-13}\right)$ or CT $\left(3.22 \pm 0.079 \mathrm{mmol} / \mathrm{L}, p=2.14 \times 10^{-11}\right)$.

After 3 months of treatments, serum TG levels were significantly decreased in the fenofibrate group and CT group relative to the serum TG before treatments ( $p=0.0024$ and $p=0.0012$, respectively, Fig. $1 \mathrm{~b}$ ), suggesting that CT decreased serum TG levels in patients with hyperlipidemia.

We next analyzed alterations of serum HDL and LDL levels and found that serum HDL did not significantly differ between before and after CT treatments (Fig. 1c), while serum LDL decreased after 3 months of treatment with CT ( $p=0.0399$, Fig. 1d). Fenofibrate can reduce serum TGand TC butdamage liver function. The two groups with hyperlipidemia patients before treatment showed an obvious increase of serum AST levels compared to healthy controls (both $p<0.001$, Fig. 1e). Administration of fenofibrate further increased serum AST levels at a 3-month follow-up ( $p$ $<0.001$ ). In contrast, CT treatment significantly decreased serum AST levels at the 3-month follow-up ( $p$ $=0.0086$, Fig. 1e).

These retrospective analyses suggested that CT could amend serum TGlevels by decreasing LDL followed by the improvement of liver function in patients with hyperlipidemia.

Table 1. Clinical baseline information in the enrolled participants. Continuous data are presented as mean \pm standard error of means (SEM) for normally distributed variables. Categorical variables are expressed as group percentages and were compared for independent samples using either Pearson $\chi 2$ or Fisher exact tests. BMI, body mass index; TG, triglyceride; HDL, high density lipoprotein; LDL, low density lipoprotein; AST, aspartate aminotransferase

\begin{tabular}{|c|c|c|c|c|}
\hline $\begin{array}{l}\text { Clinical and pathological } \\
\text { Indexes }\end{array}$ & \multirow{2}{*}{$\begin{array}{c}\text { Healthy controls } \\
(\mathrm{n}=20)\end{array}$} & \multirow{2}{*}{$\begin{array}{c}\begin{array}{c}\text { Fenofibrate } \\
(\mathrm{n}=20)\end{array} \\
46.15 \pm 1.40\end{array}$} & \multirow{2}{*}{ 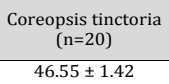 } & \multirow{2}{*}{$\begin{array}{r}\begin{array}{r}\text { P value } \\
((\text { Fen vs. } \\
\text { CT })\end{array} \\
0.842 \\
\end{array}$} \\
\hline Age (year) & & & & \\
\hline Gender & $10.0(50 \%)$ & $11(55 \%)$ & $11(55 \%)$ & 1.000 \\
\hline & $10.0(50 \%)$ & $9.0(45 \%)$ & $9.0(45 \%)$ & \\
\hline BMI $(\mathrm{kg} / \mathrm{m} 2)$ & $22.30 \pm 0.32$ & $26.55 \pm 0.55$ & $26.55 \pm 0.55$ & 1.000 \\
\hline $\mathrm{TG}(\mathrm{mmol} / \mathrm{L})$ & $1.49 \pm 0.03$ & $1.82 \pm 0.021$ & $1.82 \pm 0.018$ & 0.833 \\
\hline $\mathrm{HDL}$ (mmol/L) & $1.13 \pm 0.03$ & $0.90 \pm 0.027$ & $0.96 \pm 0.028$ & 0.097 \\
\hline $\mathrm{LDL}(\mathrm{mmol} / \mathrm{L})$ & $2.42 \pm 0.028$ & $3.27 \pm 0.073$ & $3.22 \pm 0.079$ & 0.666 \\
\hline $\operatorname{AST}(\mathrm{U} / \mathrm{L})$ & $17.70 \pm 0.236$ & $22.30 \pm 0.470$ & $24.05 \pm 0.581$ & 0.028 \\
\hline
\end{tabular}

Fig. 1. Coreopsis tinctoria amend lipid metabolism by decreasing LDL without liver injury in patients with hyperlipidemia. (a) Comparison of body mass index (BMI) for all participants in the three groups before and after the treatments for 3 months. (b) Alterations of serum triglyceride (TG) levels in the three groups before and after the treatments for 3 months. (c)\&(d) Serum levels of high density lipoprotein (HDL) and low density lipoprotein (LDL) in the three groups before and after the treatments for 3 months. (e) Alterations of serum aspartate aminotransferase (AST) levels in the three groups before and after the treatments for 3 months.

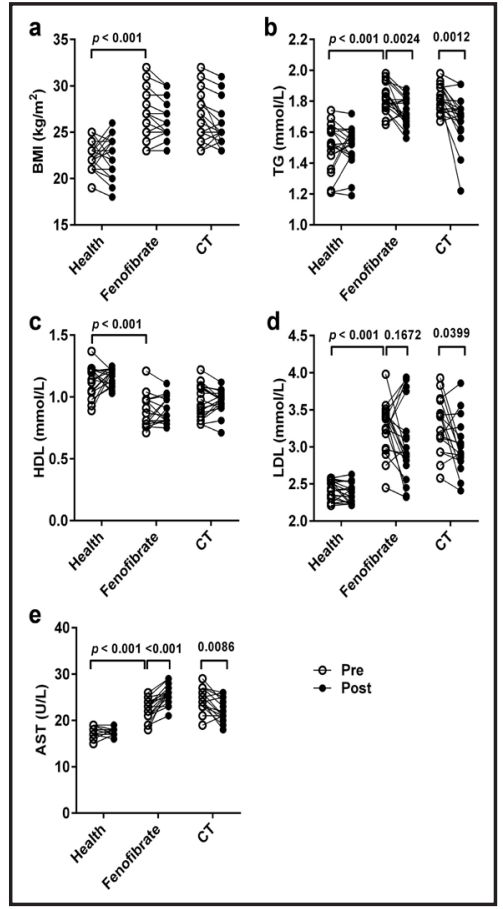


Fig. 2. Coreopsis tinctoria amend lipid metabolism without liver injury in mice with hyperlipidemia model. (a) Serum levels of triglyceride (TG), total cholesterol (TC) and low density lipoprotein (LDL) in the normal controls (NC), hyperlipidemia mice (HL) group and Coreopsis tinctoria (CT) treatment group. (b) Serum levels of total protein and albumin in the three groups. (c) Liver histology structure in the three

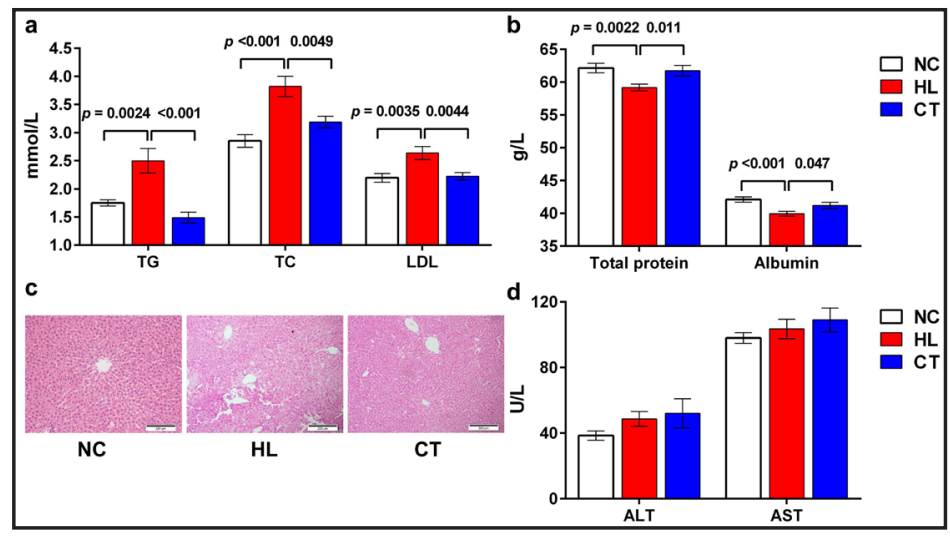
groups. (d) Serum levels of alanine aminotransferase (ALT) and aspartate aminotransferase (AST) in the three groups.

\section{CT amends lipid metabolism without liver injury in the mouse model of hyperlipidemia}

To verify the effect of CT amending lipid metabolism in patients with hyperlipidemia, we established an experimental hyperlipidemia model in mice and treated the hyperlipidemic mice with CT. Compared with NC mice, the hyperlipidemic mice presented a significant increase of serum TG and TC levels ( $p=0.0024$ and $p<0.001$, respectively), accompanied by an increase in serum LDL level ( $p=0.0035$, Fig. 2a). Importantly, oral feeding of CT remarkably decreased serum levels of TG, TC, and LDL compared with the HL group ( $p<$ $0.001, p=0.0049$, and $p=0.0044$, respectively, Fig. $2 \mathrm{a}$ ). We also noted that serum levels of total protein and albumin were decreased in the HL group relative to the NC group $(p=$ 0.0022 and $p<0.001$ ), and then were improved after CT administration relative to the HL group ( $p=0.011$ and $p=0.047$, Fig. $2 b$ ).

Moreover, we further observed live structures and function in mice with hyperlipidemia. As shown in liver histology, compared with the normal liver in the NC group, the liver in hyperlipidemic mice presented damage to liver lobular structures and an obvious steatosis of liver cells. In contrast, oral administration of CT improved liver lobular structure and attenuated liver cell steatosis (Fig. 2c). Meanwhile, there were no significant alterations among the NC, HL, or CT groups in serum ALT levels $(38.5 \pm 2.83 \mathrm{U} / \mathrm{L}, 48.7 \pm 4.47 \mathrm{U} / \mathrm{L}, 52.1$ $\pm 8.86 \mathrm{U} / \mathrm{L}$, all $p>0.05)$, or serum AST levels $(98.0 \pm 3.20 \mathrm{U} / \mathrm{L}, 103.5 \pm 5.97 \mathrm{U} / \mathrm{L}, 109.0 \pm 7.27$ $\mathrm{U} / \mathrm{L}$, all $p>0.05$ ), which indicated no damage to liver function after oral administration of CT to reduce serum lipid levels (Fig. $2 \mathrm{~d}$ ).

\section{CT improved gut microbiota composition in mice with hyperlipidemia}

The occurrence and development of cardiovascular disease was closely related to gut microbial alterations [19]. To further explore the possible mechanism of CT amending lipid metabolism, we analyzed gut microbial characteristics of mice with hyperlipidemia and identified gut microbial alterations after CT treatment using MiSeq sequencing and data analysis.

To observe sequencing depth of the data set, rarefaction curves for each group and each sample were created. The rarefaction curves among the three groups tended to be flat (Fig. $3 a$ ), indicating that the data set was sufficiently big to reflect most of the microbial diversity. A long tail was noted in the OTU-rank abundance distribution curves (Fig. 3b), suggesting that most OTUs appeared at low abundance in fecal microbiota in mice with hyperlipidemia.

Gut microbial richness and diversity were compared after equalizing sample sizes by random subtraction. Compared with normal controls, gut microbial richness and diversity, as estimated by the Shannon, Simpson, and Invsimpson indexes, were significantly decreased in mice with hyperlipidemia (all $p<0.001$, Fig. 3c). Notably, oral administration of CT increased gut microbial richness and diversity, as demonstrated by an increase in the Shannon index $(p=0.031)$. 


\section{Cellular Physiology Cell Physiol Biochem 2018;48:1060-1074 \begin{tabular}{l|l} 
DOI: 10.1159/000491973 & $\begin{array}{l}\text { O 2018 The Author(s). Published by S. Karger AG, Basel } \\
\text { www.karger.com/cpb }\end{array}$
\end{tabular}

To illustrate the similarities between different samples in bacterial communities, PCA was performed. In the weighted Unifrac PCA plot (Fig. 3d), fecal microbial communities were well separated between normal controls and the HL group according to PC1 and PC2 (45\% and $13.5 \%$ of explained variance, respectively). Notably, the bacterial community of the CT group was mildly improved and tended to approximate the normal controls. Meanwhile, the unweighted Unifrac PCA plots (Fig. 3e) also indicated a similar result. Moreover, we used another three distance analyses (Hellinger, Jensen-Shannon, and Spearman analyses, Fig. S1c) based on beta-diversity distance to verify our PCA result, and we concluded that bacterial communities were significantly separated between normal controls and the HL group and that oral administration of CT improved gut microbiota composition to that of approximately normal controls.

\section{Identification of key OTUs among the different groups}

To identify key OTUs phylotypes among the different groups, the taxonomy annotations of all OTUs were compiled. Meanwhile, the distribution and abundance of all different OTUs between normal controls and the HL group were analyzed by Wilcoxon rank-sum tests with the Benjamini-Hochberg method to correct for multiple comparisons. A total of 50 OTUs were identified as key different OTUs between normal controls and the HL group. Forty-six of these OTUs were decreased, while another four OTUs were increased in the HL group relative

Fig. 3. Coreopsistinctoriaimproved gut microbiota composition in mice with hyperlipidemia. (a) The rarefaction curves for number of reads sampled among the three groups. (b) The OUTrank abundance distribution curves among the three groups. (c) Comparisons of microbial richness and diversity, estimated by Shannon Index, Simpson Index, Invsimpson Index, Chao 1 Index, Observed species (Obs) Index, and Incidence-based Coverage Estimators (ICE) Index among the three groups. Principal coordinate analysis (PCA) of fecal microbiota

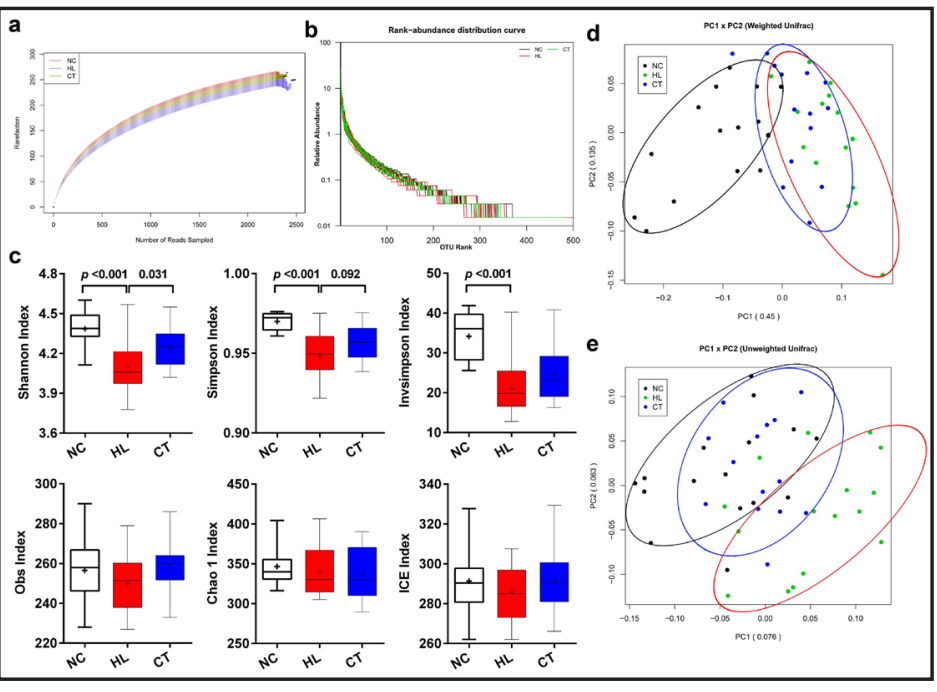
based on weighted UniFrac metric distance (d) and unweighted UniFrac metric distance (e) among the three groups were conducted.

Fig. 4. Identification of key Operational Taxonomy Units (OTUs) phylotypes among the three groups. Abundance distribution of the 50 OTUs identified as key phylotypes: 46 OTUs were decreased (black font), while 4 OTUs were enriched (red font) in the HL group versus normal controls. To show the distribution of OTUs with lower abundance, the colored squares of each row were scaled to denote the

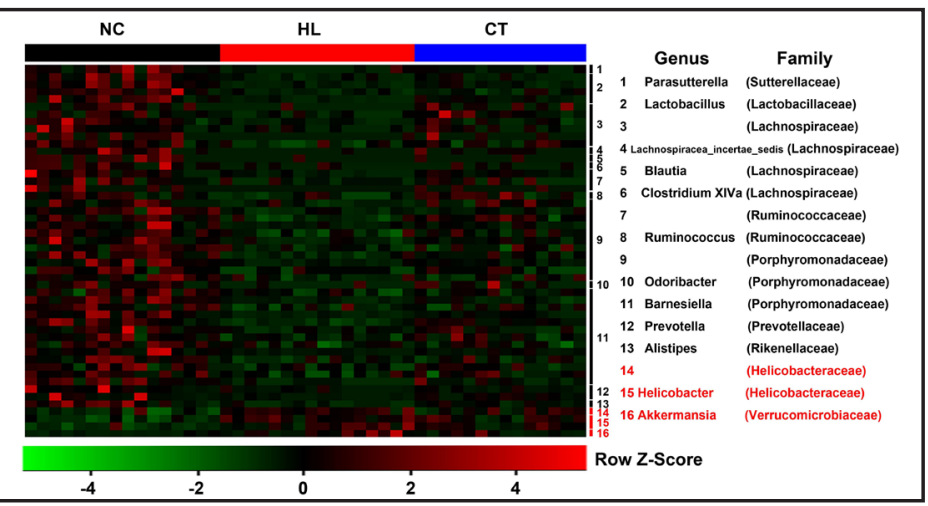
relative ratio of each OUT among the three groups. 
to the normal controls (Fig. 4). Most of these decreased OTUs were distributed across the families Porphyromonadaceae (25 OTUs), Lachnospiraceae (13 OTUs), and Ruminococcaceae (4 OTUs), while the increased OTUs were mainly distributed in the family Helicobacteraceae (3 OTUs) in the HL group versus normal controls. Importantly, after oral administration of CT, the alterations of 50 key different OTUs tended to approximate those of the normal controls. While 46 key OTUs were increased, the remaining 4 key OTUs were decreased.

\section{Taxonomy profiles of gut microbial communities among the different groups}

To illustrate the detailed bacterial changes of gut microbial communities, taxonomy profiles were analyzed among the different groups. Gut microbial communities in the three groups mainly included five bacterial phyla: Bacteroidetes, Firmicutes, Proteobacteria, Verrucomicrobia, and Deferribacteres, in which Bacteroidetes, Firmicutes, and Proteobacteria were the top three dominant populations in the three groups, together accounting for up to $90 \%$ of sequences (Fig. 5a). Compared with normal controls, the phylum Bacteroidetes was significantly decreased, while phyla Proteobacteria and Verrucomicrobia were obviously increased in the HL group ( $p<0.001$ or $p<0.01$ ). Notably, oral administration of CT attenuated the alterations of phyla Bacteroidetes, Proteobacteria, and Verrucomicrobia in the HL group (Fig. 5b).

At the class level, Bacteroidia, Clostridia, Epsilonproteobacteria, Bacilli, Erysipelotrichia, Verrucomicrobiae, Deltaproteobacteria, and Gammaproteobacteria were the top eight bacterial classes in the three groups. Compared with the normal controls, classes Bacteroidia, Bacilli, and Beta-proteobacteria were significantly decreased, while Epsilonproteobacteria, Verrucomicrobiae, and Gammaproteobacteria, corresponding to the increased phyla, were significantly increased in the HL group. Meanwhile, bacterial alterations at the order level between both groups presented a similar result (Fig. 5c,d). Bacterial communities were also analyzed at the family level. Corresponding to the dominant bacterial phyla, families Porphyromonadaceae, Lachnospiraceae, Helicobacteraceae, Bacteroidaceae, Lactobacillaceae, Prevotellaceae, Erysipelotrichaceae, Rikenellaceae, and Verrucomicrobiaceae were the predominant populations in the three groups. Compared with normal controls, the families

Fig. 5. Taxonomy profiles of gut microbial communities among the different groups. (a) Composition of gut microbiota at phylum level in the three groups. (b) Comparison of the different microbial community among the three groups at the phylum level. Compared with normal controls, the decreased microbial community (c) and the increased microbial community (d) in the HL group were shown at the class, order, family and genus levels, respectively.

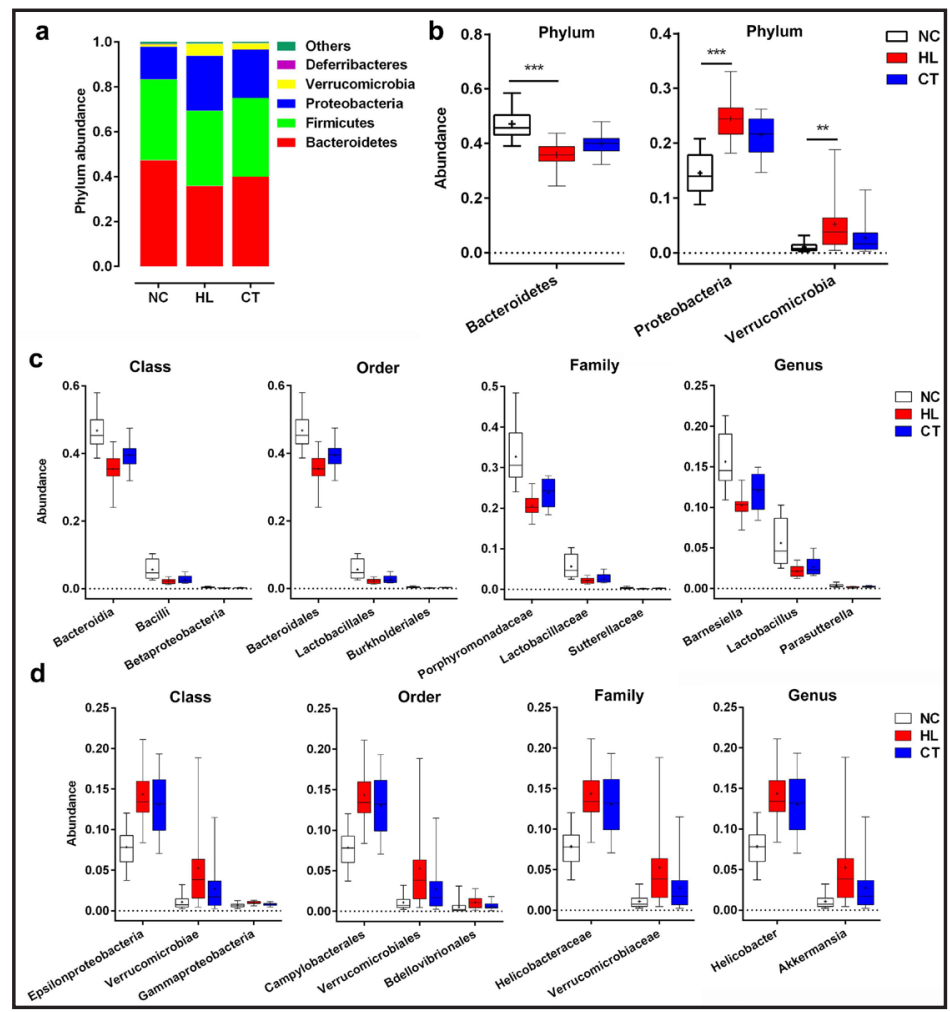




\section{$\begin{array}{lll}\text { Cellular Physiology } & \text { Cell Physiol Biochem 2018;48:1060-1074 } \\ \text { DOI: 10.1159/000491973 } & 0 \text { 2018 The Author(s). Published by S. Karger AG, Basel }\end{array}$

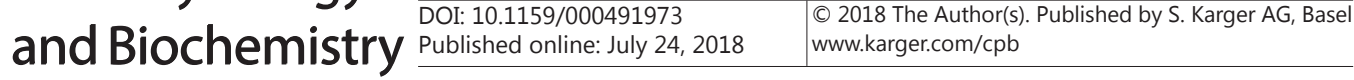 \\ Ren et al.: Coreopsis Tinctoria Modulates Lipids Metabolism}

Porphyromonadaceae, Lactobacillaceae, and Sutterellaceae were markedly decreased, while families Helicobacteraceae and Verrucomicrobiaceae were significantly increased in the HL group (Fig. 5c,d). Moreover, consistent with bacterial alterations at the family level, Barnesiella, Lactobacillus, and Parasutterella were obviously reduced, while Helicobacter and Akkermansia were significantly increased at the genus level in the HL group relative to the normal controls (Fig. 5c,d). Importantly, the oral administration of CT attenuated bacterial alterations at the class, order, family, and genus levels in the HL group (Fig. 5c,d).

Identification of the specific bacterial taxa associated with hyperlipidemia in mice using LEfSe

To identify the specific bacterial taxa associated with hyperlipidemia in mice, we analyzed gut microbiota in normal controls and the HL group using LEfSe. A cladogram representative of gut microbial structures and their predominant bacteria are shown in Fig. $6 \mathrm{a}$, and the greatest differences in taxa between the two groups are presented. Gut microbial dysbiosis was extensive in the mice with hyperlipidemia. As shown in Fig. 6b, LDA score plots display the significantly different bacterial populations among taxa and identified key phylotypes as microbial markers at different phylogenetic levels between normal controls and the HL group.

To further confirm the value of microbial markers for distinguishing hyperlipidemic mice from normal controls, a series of ROC curves of microbial markers at the genus level were constructed. The decreased bacterial genera Barnesiella and Lactobacillus in the HL group achieved very high area under the curve (AUC) values of 0.9766 and 0.9551 , respectively (Fig. 6c). Notably, the increased genus Helicobacter in the HL group also well differentiated the two groups, achieving a high AUC value of 0.9629. These data confirmed

Fig. 6. Identification of the specific bacterial taxa associated with hyperlipidemia in mice using LEfSe. (a) The LEfSe method identified the most differentially abundant taxons between normal controls and the HL group. Taxonomic cladogram obtained from LEfSe analysis of $16 \mathrm{~S}$ sequences (relative abundance $\geq$ $0.5 \%$ ). The brightness of each dot is proportional to its effect size. (b) LDA score plot displayed the significant difference bacterial populations in taxa and identified key phylotypes as microbial markers at different phylogenetic levels between normal controls and the HL group. Normal controlenriched taxa were indicated with a positive LDA score (green), and HL group-enriched taxa presented a negative score (red). Only taxa achieving an LDA significant threshold >2 were shown. (c) Receiver operating characteristic (ROC) curves of microbial markers including bacterial genus Barnesiella, Lactobacillus and Helicobacter to distinguish hyperlipidemia mice from normal controls. AUC: area under the curve.

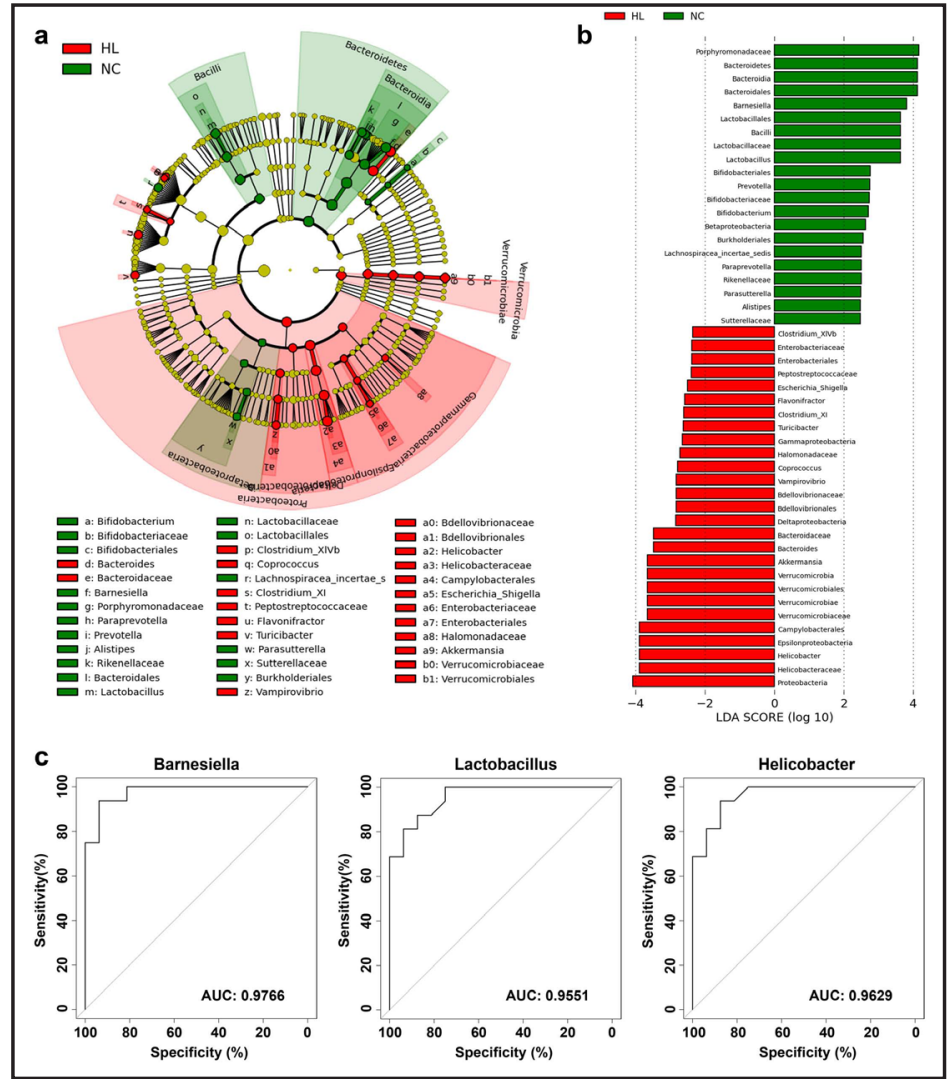


Fig. 7. Identification
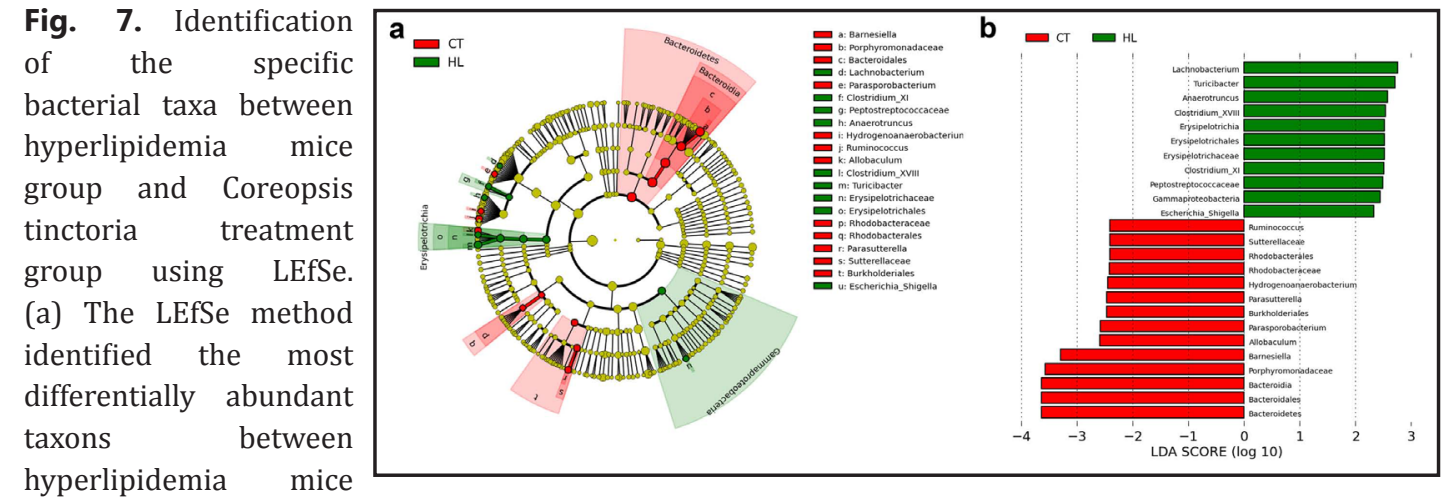

hyperlipidemia mice

group and Coreopsis

tinctoria treatment group. Taxonomic cladogram obtained from LEfSe analysis of $16 \mathrm{~S}$ sequences (relative abundance $\geq 0.5 \%$ ). The brightness of each dot is proportional to its effect size. (b) LDA score plot displayed the significant difference bacterial populations in taxa and identified key phylotypes as microbial markers at different phylogenetic levels between hyperlipidemia mice group and Coreopsis tinctoria treatment group. Hyperlipidemia -enriched taxa were indicated with a positive LDA score (green), and Coreopsis tinctoria group-enriched taxa presented a negative score (red). Only taxa achieving an LDA significant threshold $>2$ were shown.

that gut microbial biomarkers could achieve high discriminatory power in differentiating hyperlipidemic mice from normal controls.

We also further analyzed the specific bacterial taxa of gut microbiota between the HL group and CT group in mice using LEfSe. The greatest differences in taxa between the two communities are displayed in Fig. 7 a,b. In contrast to the large differences in gut microbiota between the HL group and normal controls, only a few microbial signatures in gut microbiota differed between the HL group and the CT group. Notably, Bacteroidetes was more abundant, while Gamma-proteobacteria and Erysipelotrichia were less abundant in gut microbiota in the CT group relative to the HL group. Importantly, administration of CT improved gut extensive dysbiosis and restored gut microbiota, approaching that of normal controls.

\section{Discussion}

More than 300 kinds of natural compounds have been isolated and identified from CT extracts, including over 30 flavonoids, over 30 essential mineral elements, over 20 amino acids, and dozens of aromatic compounds. CT is also rich in natural compounds with biological activity, such as organic acids, terpenes, vitamins, lignans, enzymes, and polysaccharides. Thus, many people popularly drink tea with CT on a regular basis. This study retrospectively observed the effect of CT amending lipid metabolism in patients with hyperlipidemia. We found that CT decreased serum TG and LDL without liver function damage in patients with hyperlipidemia, which was also verified in hyperlipidemic mice. Owing to the close relationship between lipid metabolism and gut microbiota, we further explored the protective role of CT on gut microbiota and proposed a novel gut microbial biomarker for hyperlipidemia.

In our previous research using an animal model, we found the active flavonoid compounds from CT extracts have anti-hyperlipidemia effects, particularly in lowering triglycerides, reducing lipid deposition, and protecting liver function [9]. Reductions in LDL can decrease major cardiovascular events in clinical trials [31,32]; thus, reducing LDL cholesterol has been incorporated into practice guidelines as a fundamental means of reducing cardiovascular morbidity and mortality $[33,34]$. In this study, serum LDL levels were significantly increased in hyperlipidemia patients before treatment with fenofibrate or CT compared with healthy controls. Importantly, serum LDL was decreased after 3 months of treatment with CT, which 


\section{Cellular Physiology Cell Physiol Biochem 2018;48:1060-1074 \\ \begin{tabular}{l|l} 
DOI: 10.1159/000491973 & $\begin{array}{l}\text { O 2018 The Author(s). Published by S. Karger AG, Basel } \\
\text { www.karger.com/cpb }\end{array}$
\end{tabular}}

Ren et al.: Coreopsis Tinctoria Modulates Lipids Metabolism

suggested that CT could amend lipid metabolism by decreasing LDL levels in patients with hyperlipidemia. As a positive control, fenofibrate was able to decrease serum TG level, but after 3 months of continuous oral intake of fenofibrate, serum AST level in patients with hyperlipidemia increased, indicating damage to liver function. In contrast, CT treatment can also decrease serum TG, LDL, and AST levels after 3 months, but without damaging liver function in patients with hyperlipidemia. In our mice study, we first found that oral administration of CT remarkably decreased serum levels of TG, TC, and LDL, but increased serum levels of total protein and albumin compared with the HL group. Liver histology indicated that oral feeding of CT improved liver lobular structure and attenuated liver cell steatosis. There were no significant alterations of serum ALT or AST levels among the three groups, although there was a slight increase in serum ALT and AST levels (within a normal range of variation $[35,36]$ ) from the $\mathrm{NC}$ group relative to the HL and CT groups. These data indicated that there was no damage to liver function after oral administration of CT to reduce serum lipid levels. CT regulated lipid metabolism by downregulating adipose differentiationrelated protein (ADRP) expression. ADRP is highly expressed in the early stage of adipose differentiation or lipid accumulation in hepatocytes [37], and it can induce increases in the number and size of lipid droplets in the liver, thereby leading to lipid accumulation and insulin signaling inhibition; thus, the decrease in ADRP could inhibit the development of fatty liver and decrease triglyceride levels in the liver. A recent study demonstrated that the flavonoidrich fraction of CT could promote glucose tolerance through pancreatic function recovery in streptozotocin-induced glucose-intolerant rats, possibly through a mechanism of action other than merely antioxidant mediation [11]. Meanwhile, CT extracts and pure compounds also showed cytoprotection that might be attributed to inhibition of the apoptotic pathway, and not through a decrease in superoxide radical production [11]. Our further studies of at least two differing doses of CT and longer consumption periods will verify the effect of differing doses of CT and longer consumption periods on lipid metabolism.

This investigation clarified the gut microbial mechanism by which CT affects lipid metabolism. The human gut microbiota maintains a symbiotic relationship with the body [12-16] and plays an important role in a series of beneficial functions including maturing the initial immune system and resistance to the invasion of enteric pathogens $[15,16]$. Moreover, gut microbiotas are associated with a spectrum of human diseases, including obesity [38], cardiovascular disease [19], type 2 diabetes (T2D) [39], inflammatory bowel diseases [40], non-alcoholic fatty liver disease [17, 18], liver cirrhosis [21], and hepatocellular carcinoma $[41,42]$. Notably, hyperlipidemia is closely associated with the occurrence and development of obesity, cardiovascular diseases [33], T2D, and non-alcoholic fatty liver disease [1, 2]; thus, we speculated that the occurrence of hyperlipidemia and the improvement of lipid metabolism were closely related to gut microbiota alterations. Human intestinal microbial richness and diversity were closely associated with health and disease [43]. There were significant intersubject variability and differences in stool microbial composition and diversity, which might elucidate the role of microbial diversity in health and disease [12]. The healthy populations were always associated with increased richness and diversity of gut microbiota. Gut microbial diversity was influenced by multiple factors, in which diet was an important factor. Diet-induced extinction of gut microbial taxa and diversity may be impacted for generations [44]. Our results demonstrated that gut microbial richness and diversity were significantly decreased in mice with hyperlipidemia, but oral administration of CT increased gut microbial richness and diversity. Importantly, bacterial communities were significantly separated between normal controls and hyperlipidemic mice, but oral administration of CT improved gut microbiota composition to approximately reflect that of normal controls.

Bacteroidetes, Firmicutes, Proteobacteria, and Verrucomicrobia were the top dominant populations in the three groups, together accounting for up to $95 \%$ of sequences, in which the abundance and relationship between Bacteroidetes and Firmicutes significantly contributed to gut microbial composition and changes $[45,46]$. Notably, we found that phylum Bacteroidetes were decreased, while phyla Proteobacteria and Verrucomicrobia 


\section{Cellular Physiology Cell Physiol Biochem 2018;48:1060-1074 \\ \begin{tabular}{c|c} 
DOI: 10.1159/000491973 & O 2018 The Author(s). Published by S. Karger AG, Basel \\
www.karger.com/cpb
\end{tabular}}

Ren et al.: Coreopsis Tinctoria Modulates Lipids Metabolism

were increased in the HL and CT groups relative to normal controls, although the proportion of Firmicutes exhibited no significant alterations among the three groups. Genera Barnesiella, Lactobacillus, and Parasutterella were obviously reduced, while Helicobacter and Akkermansia were significantly increased in the HL group relative to normal controls. CT oral administration attenuated bacterial composition alterations in the HL group. However, in recent studies, the improvement in cardiometabolic risk markers following a multifunctional diet was associated with gut microbial taxa in relatively healthy overweight and obese subjects, but dietary interventions did not significantly alter the gut microbiota composition at the phylum or genus levels [47]. Also, compared with a refined grain diet, whole grain diets did not alter insulin sensitivity and gut microbiomes but did reduce body weight and systemic low-grade inflammation [48].

There is a striking finding that gut microbial biomarkers can discriminate hyperlipidemia from normal controls. Qin et al [49]. carried out gut microbial analysis in T2D patients and created an accurate classifier for the diagnosis of T2D. Soon afterwards, Qin et al [23]. characterized the gut microbiome of individuals with liver cirrhosis and established an accurate patient discrimination index based on only 15 microbial biomarkers. Our recent study also demonstrated that based on 40 genera associated with pancreatic carcinoma, gut microbial markers achieve high classification power from 16S rRNA MiSeq sequencing data [50]. In our mice model with hyperlipidemia, a cladogram representative of gut microbiota and LDA score plot displayed significant differentiation of bacterial populations in taxa and identified key phylotypes as microbial markers. Importantly, the genera Barnesiella, Lactobacillus, and Helicobacter achieved very high AUC values of 0.9766, 0.9551, and 0.9629, respectively, to distinguish hyperlipidemic mice from normal controls. Thus, our data further supported that gut microbial biomarkers could achieve a high discrimination power with respect to hyperlipidemia.

In conclusion, CT modulated blood lipid metabolism by decreasing LDL without liver function damage in patients with hyperlipidemia. An animal model of hyperlipidemia also indicated that CT amended lipid metabolism without liver injury. Gut microbial richness and diversity were significantly decreased in mice with hyperlipidemia, but oral administration of CT increased microbial richness and diversity. Bacterial communities were significantly differentiated between normal controls and hyperlipidemic mice, but oral feeding of CT improved gut microbiota composition to approximate those of normal controls. Meanwhile, oral administration of CT attenuated bacterial alterations at the class, order, family, and genus levels in hyperlipidemic mice. Importantly, gut microbial biomarkers achieved a high discriminatory power in differentiating hyperlipidemic mice from normal controls. These findings may provide novel therapeutic strategies for patients with hyperlipidemia.

\section{Abbreviations}

CT (Coreopsis tinctoria); LDL (low density lipoprotein); TG (Triglyceride); LDL (low density lipoprotein); BMI (body mass index); HL (Hyperlipidemia); NC (normal control); TC (total cholesterol); TG (triglyceride); HDL (high density lipoprotein); LDL (low density lipoprotein); ALT (alanine aminotransferase); AST (aspartate aminotransferase); H\&E (hematoxylin and eosin); OTUs (Operational Taxonomy Units); PCA (Principal Component Analysis); LDA (linear discriminant analysis); LEfSe (LDA effect size); SEM (standard error of means); ROC (receiver operating characteristics); AUC (area under the curve); T2D (type 2 diabetes); PDI (patient discrimination index).

\section{Acknowledgements}

This study was sponsored by grants from National S\&T Major Project of China (2018ZX10301201), National Natural Science Foundation of China $(81600506,81702757$ and 81702346), China Postdoctoral Science Foundation (2017464 and 20182814), Xinjiang Natural Science Foundation (2015211C053), Youth innovation fund of First Affiliated Hospital of Zhengzhou University (YNQN2017032 and YNQN2017031), Joint research fund 
of First Affiliated Hospital of Zhengzhou University and Dalian Institute of Chemical Physics, Chinese Academy of Sciences (RZG and SRR). The funding sources had no role in the design of this study nor any role during its execution, analyses, data interpretation, or decision to submit results. We thank Dr. Jie Xue from Soochow University to help sample collection, and thank Dr. Hongyan Ren from Shanghai Itechgene Technology Co. Ltd., China.

The raw Illumina read data for all samples have been deposited in the European Bioinformatics Institute European Nucleotide Archive database (Study accession Number: PRJEB15125, Secondary study accession number: ERP016828).

The study was approved by the Institutional Review Board of the First Affiliated Hospital of Zhengzhou University and the First Affiliated Hospital, Xinjiang Medical University. All participants provided a written informed consent upon enrolment.

XC and ZY designed the study. ZR, JL and LH performed animal study; YL, HL and MW completed clinical study; GC, JS and YS extracted DNA. AL and RS finished sequencing and data analysis; ZR and XC wrote the manuscript. All authors reviewed and approved the manuscript.

\section{Disclosure Statement}

The authors declare that they have no competing interests.

\section{References}

1 Ng R, Wu H, Xiao H, Chen X, Willenbring H, Steer CJ, Song G: Inhibition of microRNA-24 expression in liver prevents hepatic lipid accumulation and hyperlipidemia. Hepatology 2014;60:554-564.

2 Calle EE, Kaaks R: Overweight, obesity and cancer: epidemiological evidence and proposed mechanisms. Nat Rev Cancer 2004;4:579-591.

- 3 Punekar RS, Fox KM, Richhariya A, Fisher MD, Cziraky M, Gandra SR, Toth PP: Burden of First and Recurrent Cardiovascular Events Among Patients With Hyperlipidemia. Clin Cardiol 2015;38:483-491.

4 Pursnani A, Massaro JM, D’Agostino RB, Sr., O’Donnell CJ, Hoffmann U: Guideline-Based Statin Eligibility, Coronary Artery Calcification, and Cardiovascular Events. JAMA 2015;314:134-141.

5 Saini M, Suministrado MS, Hilal S, Dong YH, Venketasubramanian N, Ikram MK, Chen C: Prevalence and Risk Factors of Acute Incidental Infarcts. Stroke 2015;46:2722-2727.

6 Rahimi K, Majoni W, Merhi A, Emberson J: Effect of statins on ventricular tachyarrhythmia, cardiac arrest, and sudden cardiac death: a meta-analysis of published and unpublished evidence from randomized trials. Eur Heart J 2012;33:1571-1581.

7 Brunt EM, Janney CG, Di Bisceglie AM, Neuschwander-Tetri BA, Bacon BR: Nonalcoholic steatohepatitis: a proposal for grading and staging the histological lesions. Am J Gastroenterol 1999;94:2467-2474.

8 Scott R, O’Brien R, Fulcher G, Pardy C, D’Emden M, Tse D, Taskinen MR, Ehnholm C, Keech A, Fenofibrate I, Event Lowering in Diabetes Study I: Effects of fenofibrate treatment on cardiovascular disease risk in 9, 795 individuals with type 2 diabetes and various components of the metabolic syndrome: the Fenofibrate Intervention and Event Lowering in Diabetes (FIELD) study. Diabetes Care 2009;32:493-498.

-9 Li Y, Chen X, Xue J, Liu J, Chen X, Wulasihan M: Flavonoids furom Coreopsis tinctoria adjust lipid metabolism in hyperlipidemia animals by down-regulating adipose differentiation-related protein. Lipids Health Dis 2014;13:193.

10 Dias T, Bronze MR, Houghton PJ, Mota-Filipe H, Paulo A: The flavonoid-rich fraction of Coreopsis tinctoria promotes glucose tolerance regain through pancreatic function recovery in streptozotocin-induced glucose-intolerant rats. J Ethnopharmacol 2010;132:483-490.

11 Dias T, Liu B, Jones P, Houghton PJ, Mota-Filipe H, Paulo A: Cytoprotective effect of Coreopsis tinctoria extracts and flavonoids on tBHP and cytokine-induced cell injury in pancreatic MIN6 cells. J Ethnopharmacol 2012;139:485-492.

12 Eckburg PB, Bik EM, Bernstein CN, Purdom E, Dethlefsen L, Sargent M, Gill SR, Nelson KE, Relman DA: Diversity of the human intestinal microbial flora. Science 2005;308:1635-1638. 


\section{Cellular Physiology Cell Physiol Biochem 2018;48:1060-1074 \begin{tabular}{l|l} 
DOI: 10.1159/000491973 & $\begin{array}{l}\text { O 2018 The Author(s). Published by S. Karger AG, Basel } \\
\text { www.karger.com/cpb }\end{array}$
\end{tabular}}

Ren et al.: Coreopsis Tinctoria Modulates Lipids Metabolism

13 Nenci A, Becker C, Wullaert A, Gareus R, van Loo G, Danese S, Huth M, Nikolaev A, Neufert C, Madison B, Gumucio D, Neurath MF, Pasparakis M: Epithelial NEMO links innate immunity to chronic intestinal inflammation. Nature 2007;446:557-561.

14 Cani PD, Delzenne NM: The role of the gut microbiota in energy metabolism and metabolic disease. Curr Pharm Des 2009;15:1546-1558.

15 Ley RE, Peterson DA, Gordon JI: Ecological and evolutionary forces shaping microbial diversity in the human intestine. Cell 2006;124:837-848.

16 Backhed F, Ley RE, Sonnenburg JL, Peterson DA, Gordon JI: Host-bacterial mutualism in the human intestine. Science 2005;307:1915-1920.

17 Le Roy T, Llopis M, Lepage P, Bruneau A, Rabot S, Bevilacqua C, Martin P, Philippe C, Walker F, Bado A, Perlemuter G, Cassard-Doulcier AM, Gerard P: Intestinal microbiota determines development of nonalcoholic fatty liver disease in mice. Gut 2013;62:1787-1794.

18 Zhu L, Baker SS, Gill C, Liu W, Alkhouri R, Baker RD, Gill SR: Characterization of gut microbiomes in nonalcoholic steatohepatitis (NASH) patients: a connection between endogenous alcohol and NASH. Hepatology 2013;57:601-609.

19 Wang Z, Klipfell E, Bennett BJ, Koeth R, Levison BS, Dugar B, Feldstein AE, Britt EB, Fu X, Chung YM, Wu Y, Schauer P, Smith JD, Allayee H, Tang WH, DiDonato JA, Lusis AJ, Hazen SL: Gut flora metabolism of phosphatidylcholine promotes cardiovascular disease. Nature 2011;472:57-63.

20 Ren Z, Jiang J, Lu H, Chen X, He Y, Zhang H, Xie H, Wang W, Zheng S, Zhou L: Intestinal microbial variation may predict early acute rejection after liver transplantation in rats. Transplantation 2014;98:844-852.

-21 Chen Y, Yang F, Lu H, Wang B, Lei D, Wang Y, Zhu B, Li L: Characterization of fecal microbial communities in patients with liver cirrhosis. Hepatology 2011;54:562-572.

-22 Ren Z, Cui G, Lu H, Chen X, Jiang J, Liu H, He Y, Ding S, Hu Z, Wang W, Zheng S: Liver ischemic preconditioning (IPC) improves intestinal microbiota following liver transplantation in rats through $16 \mathrm{~s}$ rDNA-based analysis of microbial structure shift. PLoS One 2013;8:e75950.

23 Qin N, Yang F, Li A, Prifti E, Chen Y, Shao L, Guo J, Le Chatelier E, Yao J, Wu L, Zhou J, Ni S, Liu L, Pons N, Batto JM, Kennedy SP, Leonard P, Yuan C, Ding W, Hu X, Zheng B, Qian G, Xu W, Ehrlich SD, Zheng S, Li L: Alterations of the human gut microbiome in liver cirrhosis. Nature 2014;513:59-64.

24 Magoc T, Salzberg SL: FLASH: fast length adjustment of short reads to improve genome assemblies. Bioinformatics 2011;27:2957-2963.

25 Edgar RC, Haas BJ, Clemente JC, Quince C, Knight R: UCHIME improves sensitivity and speed of chimera detection. Bioinformatics 2011;27:2194-2200.

-26 Edgar RC: UPARSE: highly accurate OTU sequences from microbial amplicon reads. Nat Methods 2013;10:996-998.

27 Wang Q, Garrity GM, Tiedje JM, Cole JR: Naive Bayesian classifier for rapid assignment of rRNA sequences into the new bacterial taxonomy. Appl Environ Microbiol 2007;73:5261-5267.

28 McMurdie PJ, Holmes S: phyloseq: an R package for reproducible interactive analysis and graphics of microbiome census data. PLoS One 2013;8:e61217.

-29 Segata N, Izard J, Waldron L, Gevers D, Miropolsky L, Garrett WS, Huttenhower C: Metagenomic biomarker discovery and explanation. Genome Biol 2011;12:R60.

30 Ling Z, Liu X, Jia X, Cheng Y, Luo Y, Yuan L, Wang Y, Zhao C, Guo S, Li L, Xu X, Xiang C: Impacts of infection with different toxigenic Clostridium difficile strains on faecal microbiota in children. Sci Rep 2014;4:7485.

31 Baigent C, Keech A, Kearney PM, Blackwell L, Buck G, Pollicino C, Kirby A, Sourjina T, Peto R, Collins R, Simes R, Cholesterol Treatment Trialists C: Efficacy and safety of cholesterol-lowering treatment: prospective meta-analysis of data from 90, 056 participants in 14 randomised trials of statins. Lancet 2005;366:1267-1278.

-32 Cholesterol Treatment Trialists C, Baigent C, Blackwell L, Emberson J, Holland LE, Reith C, Bhala N, Peto R, Barnes EH, Keech A, Simes J, Collins R: Efficacy and safety of more intensive lowering of LDL cholesterol: a meta-analysis of data from 170, 000 participants in 26 randomised trials. Lancet 2010;376:1670-1681.

-33 Anderson TJ, Gregoire J, Hegele RA, Couture P, Mancini GB, McPherson R, Francis GA, Poirier P, Lau DC, Grover S, Genest J, Jr., Carpentier AC, Dufour R, Gupta M, Ward R, Leiter LA, Lonn E, Ng DS, Pearson GJ, Yates GM, Stone JA, Ur E: 2012 update of the Canadian Cardiovascular Society guidelines for the diagnosis and treatment of dyslipidemia for the prevention of cardiovascular disease in the adult. Can J Cardiol 2013;29:151-167. 


\section{Cellular Physiology Cell Physiol Biochem 2018;48:1060-1074 \begin{tabular}{l|l} 
DOI: 10.1159/000491973 & $\begin{array}{l}\text { O 2018 The Author(s). Published by S. Karger AG, Basel } \\
\text { www.karger.com/cpb }\end{array}$
\end{tabular}}

Ren et al.: Coreopsis Tinctoria Modulates Lipids Metabolism

34 Sabatine MS, Giugliano RP, Wiviott SD, Raal FJ, Blom DJ, Robinson J, Ballantyne CM, Somaratne R, Legg J, Wasserman SM, Scott R, Koren MJ, Stein EA, Open-Label Study of Long-Term Evaluation against LDLCI: Efficacy and safety of evolocumab in reducing lipids and cardiovascular events. N Engl J Med 2015;372:1500-1509.

-35 Sun P, Zeng Q Cheng D, Zhang K, Zheng J, Liu Y, Yuan YF, Tang YD: Caspase Recruitment Domain Protein 6 protects against hepatic steatosis and insulin resistance by suppressing Ask1. Hepatology 2018;10.

-36 Li N, Dou Z, Liu J, Chai B, Li Y, An X, Chu P, Zhang X: Therapeutic Effect of HGF on NASH Mice Through HGF/ c-Met and JAK2-STAT3 Signalling Pathway. Ann Hepatol 2018;17:501-510.

-37 Grasselli E, Voci A, Pesce C, Canesi L, Fugassa E, Gallo G, Vergani L: PAT protein mRNA expression in primary rat hepatocytes: Effects of exposure to fatty acids. Int J Mol Med 2010;25:505-512. Zhao L: The gut microbiota and obesity: from correlation to causality. Nat Rev Microbiol 2013;11:639-647. Qin J, Li Y, Cai Z, Li S, Zhu J, Zhang F, Liang S, Zhang W, Guan Y, Shen D, Peng Y, Zhang D, Jie Z, Wu W, Qin Y, Xue W, Li J, Han L, Lu D, Wu P, Dai Y, Sun X, Li Z, Tang A, Zhong S, Li X, Chen W, Xu R, Wang M, Feng Q, Gong M, Yu J, Zhang Y, Zhang M, Hansen T, Sanchez G, Raes J, Falony G, Okuda S, Almeida M, LeChatelier E, Renault P, Pons N, Batto JM, Zhang Z, Chen H, Yang R, Zheng W, Yang H, Wang J, Ehrlich SD, Nielsen R, Pedersen $\mathrm{O}$, Kristiansen K: A metagenome-wide association study of gut microbiota in type 2 diabetes. Nature 2012;490:55-60.

40 Sartor RB: Microbial influences in inflammatory bowel diseases. Gastroenterology 2008;134:577-594.

41 Dapito DH, Mencin A, Gwak GY, Pradere JP, Jang MK, Mederacke I, Caviglia JM, Khiabanian H, Adeyemi A, Bataller R, Lefkowitch JH, Bower M, Friedman R, Sartor RB, Rabadan R, Schwabe RF: Promotion of hepatocellular carcinoma by the intestinal microbiota and TLR4Cancer Cell 2012;21:504-516.

-42 Lu H, Ren Z, Li A, Zhang H, Jiang J, Xu S, Luo Q, Zhou K, Sun X, Zheng S, Li L: Deep sequencing reveals microbiota dysbiosis of tongue coat in patients with liver carcinoma. Sci Rep 2016;6:33142.

-43 Lynch SV, Pedersen 0: The Human Intestinal Microbiome in Health and Disease. N Engl J Med 2016;375:2369-2379.

44 Sonnenburg ED, Smits SA, Tikhonov M, Higginbottom SK, Wingreen NS, Sonnenburg JL: Diet-induced extinctions in the gut microbiota compound over generations. Nature 2016;529:212-215.

45 Qin N, Yang F, Li A, Prifti E, Chen Y, Shao L, Guo J, Le Chatelier E, Yao J, Wu L, Zhou J, Ni S, Liu L, Pons N, Batto JM, Kennedy SP, Leonard P, Yuan C, Ding W, Chen Y, Hu X, Zheng B, Qian G, Xu W, Ehrlich SD, Zheng S, Li L: Alterations of the human gut microbiome in liver cirrhosis. Nature 2014;513:59-64.

46 Ren Z, Jiang J, Xie H, Li A, Lu H, Xu S, Zhou L, Zhang H, Cui G, Chen X, Liu Y, Wu L, Qin N, Sun R, Wang W, Li L, Wang W, Zheng S: Gut microbial profile analysis by MiSeq sequencing of pancreatic carcinoma patients in China. Oncotarget 2017;8:95176-95191.

47 Marungruang N, Tovar J, Bjorck I, Hallenius FF: Improvement in cardiometabolic risk markers following a multifunctional diet is associated with gut microbial taxa in healthy overweight and obese subjects. Eur J Nutr 2017;10.

-48 Roager HM, Vogt JK, Kristensen M, Hansen LBS, Ibrugger S, Maerkedahl RB, Bahl MI, Lind MV, Nielsen RL, Frokiaer H, Gobel RJ, Landberg R, Ross AB, Brix S, Holck J, Meyer AS, Sparholt MH, Christensen AF, Carvalho V, Hartmann B, Holst JJ, Rumessen JJ, Linneberg A, Sicheritz-Ponten T, Dalgaard MD, Blennow A, Frandsen HL, Villas-Boas S, Kristiansen K, Vestergaard H, Hansen T, Ekstrom CT, Ritz C, Nielsen HB, Pedersen OB, Gupta R, Lauritzen L, Licht TR: Whole grain-rich diet reduces body weight and systemic low-grade inflammation without inducing major changes of the gut microbiome: a randomised cross-over trial. Gut 2017. Doi:10.1136/gutjnl-2017-314786

-49 Qin J, Li Y, Cai Z, Li S, Zhu J, Zhang F, Liang S, Zhang W, Guan Y, Shen D, Peng Y, Zhang D, Jie Z, Wu W, Qin Y, Xue W, Li J, Han L, Lu D, Wu P, Dai Y, Sun X, Li Z, Tang A, Zhong S, Li X, Chen W, Xu R, Wang M, Feng Q, Gong M, Yu J, Zhang Y, Zhang M, Hansen T, Sanchez G, Raes J, Falony G, Okuda S, Almeida M, LeChatelier E, Renault P, Pons N, Batto JM, Zhang Z, Chen H, Yang R, Zheng W, Li S, Yang H, Wang J, Ehrlich SD, Nielsen R, Pedersen O, Kristiansen K, Wang J: A metagenome-wide association study of gut microbiota in type 2 diabetes. Nature 2012;490:55-60.

50 Ren Z, Jiang J, Xie H, Li A, Lu H, Xu S, Zhou L, Zhang H, Cui G, Chen X, Liu Y, Wu L, Qin N, Sun R, Wang W, Li L, Wang W, Zheng S: Gut microbial profile analysis by MiSeq sequencing of pancreatic carcinoma patients in China. Oncotarget 2017;8:95176-95191. 